\title{
Rapid Determination of the Monosaccharide Composition and Contents in Tea Polysaccharides from Yingshuang Green Tea by Pre-Column Derivatization HPLC
}

\author{
Yujie Ai, Zhi Yu, Yuqiong Chen, Xiaojing Zhu, Zeyi Ai, Shuyuan Liu, and Dejiang Ni \\ Key Laboratory of Horticultural Plant Biology, Ministry of Education, College of Horticulture and Forestry Sciences, \\ Huazhong Agricultural University, Wuhan 430070, China \\ Correspondence should be addressed to Dejiang Ni; nidj@mail.hzau.edu.cn
}

Received 18 April 2016; Accepted 11 August 2016

Academic Editor: Somdet Srichairatanakool

Copyright ( 2016 Yujie Ai et al. This is an open access article distributed under the Creative Commons Attribution License, which permits unrestricted use, distribution, and reproduction in any medium, provided the original work is properly cited.

\begin{abstract}
A pre-column derivatization high-performance liquid chromatography (HPLC) method was developed and optimized to characterize and quantify the monosaccharides present in tea polysaccharides (TPS) isolated from Yingshuang green tea. TPS sample was hydrolyzed with trifluoroacetic acid, subjected to pre-column derivatization using 1-phenyl-3-methyl-5-pyrazolone (PMP), and separated on an Agilent TC- $\mathrm{C}_{18}$ column $(4.6 \mathrm{~mm} \times 250 \mathrm{~mm}, 5 \mu \mathrm{m})$ with UV detection at $250 \mathrm{~nm}$. A mixture of ten PMP derivatives of standard monosaccharides (mannose, ribose, rhamnose, glucuronic acid, galacturonic acid, glucose, xylose, galactose, arabinose, and fucose) could be baseline separated within $20 \mathrm{~min}$. Moreover, quantitative analysis of the component monosaccharides in Yingshuang green tea TPS was achieved, indicating the TPS consisted of mannose, ribose, rhamnose, glucuronic acid, galacturonic acid, glucose, xylose, galactose, and arabinose in the molar contents of $0.72,0.78,0.89,0.13,0.15$, $0.36,0.39,0.36,0.36$, and $0.38 \mu \mathrm{M}$, respectively. Recovery efficiency for component monosaccharides from TPS ranged from 93.6 to $102.4 \%$ with RSD values lower than $2.5 \%$. In conclusion, pre-column derivatization HPLC provides a rapid, reproducible, accurate, and quantitative method for analysis of the monosaccharide composition and contents in TPS, which may help to further explore the relationship between TPS monosaccharides isolated from different tea varieties and their biological activity.
\end{abstract}

\section{Introduction}

Tea, coffee, and cocoa are the three major nonalcoholic beverages consumed throughout the world. Crude tea is commonly used to treat diabetes mellitus in Chinese and Japanese traditional medicine, and TPS have been identified as the pharmacologically active ingredients in crude tea. Recent pharmacological studies indicated TPS may help to prevent and treat cardiovascular disease [1], possess antioxidative [2], anticancer [3], anticoagulative, and antiatherosclerotic activities $[4,5]$, exert hypoglycemic and bradycardic effects $[6,7]$, and enhance nonspecific immunological function [8]. Therefore, the components of TPS may have the potential to treat a variety of conditions.

TPS are a range of complex glycoproteins, and their biological activity is related to their primary structure. TPS composed of arabinose, ribose, and galactose demonstrated the highest ability to reduce bloody fat [9], whereas TPS are composed primarily of galactose and glucose demonstrated hypoglycemic activity [10]. In addition, our laboratory reported that TPS isolated from different tea varieties had varied antioxidant capacities, with TPS from Yingshuang green tea showing the highest antioxidant activity $[11,12]$. Therefore, it is vitally important to characterize the monosaccharide and polysaccharide compositions of TPS isolated from different tea varieties in order to investigate the relationship between the structure and composition of TPS and their antioxidant capacity and biological activities in more detail.

As analytical methods for sugars have developed, the main chromatographic methods currently used to analyze monosaccharides are gas chromatography (GC) [13], thinlayer chromatography (TLC) [14], and HPLC [15]. However, each of these techniques possesses a number of disadvantages. As monosaccharides are nonvolatile, monosaccharide molecules need to be converted into volatile derivatives with high thermal stability via a complicated process in order 
to be analyzed by GC. TLC is subject to the unresolved issues of poor reproducibility and poor color rendering for microconstituents. HPLC with a carbohydrate analysis column or NH2 column are commonly used for monosaccharide analysis; however, both techniques are expensive. Additionally, derivative isomers of monosaccharides can be generated during HPLC, which produces other peaks and affects the separation and quantitative analysis of sugars. Moreover, as monosaccharides do not absorb ultraviolet light, pre- or post-column derivatization is required in order to improve the separation selectivity and detection sensitivity of HPLC. The PMP pre-column derivation HPLC method uses mild reaction conditions, does not result in stereoisomerism products and has a high detection sensitivity, and is used more widely than other derivation methods $[16,17]$.

In recent years, pre-column derivatization HPLC has been used to successfully separate mixtures of ten monosaccharides (Rha, Ara, Xyl, Man, Glc, Gal, Fuc, Rib, GlcA, and GalA); this analysis can be accomplished within 40 to $30 \mathrm{~min}$ $[18,19]$, though all of reported methods are time consuming and inaccurate. In order to develop a more rapid and accurate method for analysis of the monosaccharides present in TPS, in this study we developed and optimized a pre-column PMP derivatization HPLC method using a Diode Array Detector (DAD) at $250 \mathrm{~nm}$ to simultaneously separate ten monosaccharides (aldoses and uronic acids). Moreover, the pre-column PMP derivatization HPLC method was validated by performing quantitative analysis of the composition and concentrations of monosaccharides in TPS isolated from Yingshuang green tea.

\section{Materials and Methods}

2.1. Materials and Reagents. Yingshuang green tea was purchased from the Tea Research Institute, Chinese Academy of Agricultural Sciences, Zhejiang province, China; Dmannose, $D$-ribose, $L$-rhamnose, $D$-glucose, $D$-xylose, $D$ galactose, $L$-arabinose, $D$-glucuronic acid, $D$-galacturonic acid, and $D$-fucose were obtained from Sigma (St. Louis, MO, USA); trifluoroacetic acid (TFA) was purchased from Sinopharm (Shanghai, China); PMP was obtained from Merck (Darmstadt, Germany) and recrystallized three times from chromatographic-grade methanol before use. HPLCgrade methanol and acetonitrile were purchased from TEDIA (Fair Lawn, NJ, USA); triethylamine was purchased from Sinopharm. All other chemicals were of analytical purity. All aqueous solutions were prepared using freshly prepared double-distilled water.

2.2. Extraction of TPS from Yingshuang Green Tea. Powdered Yingshuang green tea $(100 \mathrm{~g})$ was extracted with distilled water $(1: 20, \mathrm{w} / \mathrm{v})$ at $55^{\circ} \mathrm{C}$ for $2 \mathrm{~h}$. The extract was centrifuged at $4000 \mathrm{rpm}$ for $15 \mathrm{~min}$ to remove contaminants, and the supernatant was precipitated with three volumes of $95 \%$ ethanol, centrifuged at $4000 \mathrm{rpm}$ for $15 \mathrm{~min}$, and washed thrice with petroleum ether, acetone, and pure ethanol. The polysaccharide pellets were dissolved in an appropriate volume of distilled water and intensively dialyzed for three days against distilled water (Mw cut-off $8000 \mathrm{Da})$. Proteins were removed by repeating the Sevag method six times [20, 21] and lyophilized as TP (1.6 g) using a freeze-dry apparatus (HO10-0035, Labogene Co., Denmark).

2.3. Hydrolysis of TPS. The TPS sample $(10 \mathrm{mg})$ was dissolved in $1 \mathrm{~mL}$ of $3 \mathrm{M}$ trifluoroacetic acid in a $5 \mathrm{~mL}$ ampoule, incubated at $130^{\circ} \mathrm{C}$ for $2 \mathrm{~h}$, the cooled reaction mixture was centrifuged at $2000 \mathrm{rpm}$ for $5 \mathrm{~min}$ and evaporated to dryness under reduced pressure to remove TFA, and the hydrolyzed and dried samples were redissolved in $1 \mathrm{~mL}$ of distilled water for the following experiments.

2.4. Derivatization of Hydrolyzed TPS with PMP. The hydrolyzed TPS sample was labeled by adding $30 \mu \mathrm{L}$ of $\mathrm{NaOH}$ $(0.3 \mathrm{M})$ and $20 \mu \mathrm{L}$ of PMP solution $(0.5 \mathrm{M}$ in methanol). Fucose was added as an internal standard to each sample before derivatization. The mixtures were incubated at $70^{\circ} \mathrm{C}$ for $60 \mathrm{~min}$, cooled to room temperature, and neutralized with $30 \mu \mathrm{L}$ of $\mathrm{HCl}(0.3 \mathrm{M}), 1 \mathrm{~mL}$ of trichloromethane was added, and, after vigorous shaking and layering, the organic phase was carefully removed and discarded. The aqueous layer was passed through a $0.45 \mu \mathrm{m}$ syringe filter before HPLC analysis. Standard solutions of the ten monosaccharides (Rha, Ara, Xyl, Man, Glc, Gal, Fuc, Rib, GlcA, and GalA; $0.1 \mu \mathrm{M}$ ) were also treated as described above.

2.5. PMP-HPLC-DAD Analysis. The PMP-labeled monosaccharides were analyzed using an Agilent 1260 HPLC system (Waldbronn, Germany) consisting of a G1311C Quaternary pump, G1329B autosampler $(0.1-100 \mu \mathrm{L}), \mathrm{G} 1316 \mathrm{~A}$ column oven (273-333 K), and G1315D-DAD detector (190-950 nm). The analytical column was a $\mathrm{TC}-\mathrm{C}_{18}$ column $(4.6 \mathrm{~mm} \times$ $250 \mathrm{~mm}, 5 \mu \mathrm{m}$; Agilent). The injection volume was $20 \mu \mathrm{L}$ with an eluant flow rate of $1.0 \mathrm{~mL} / \mathrm{min}$ at $35^{\circ} \mathrm{C}$. Mobile phase $\mathrm{A}$ was $100 \%$ acetonitrile and mobile phase $\mathrm{B}$ was a mixture of distilled water and acetonitrile $\left(90: 10\right.$, v/v) with $0.045 \% \mathrm{KH}_{2}$ $\mathrm{PO}_{4}-0.05 \%$ triethylamine buffer $(\mathrm{pH} 7.5)$; gradient elution was performed at 94-94-88-88\% B with linear decreases at 04-5-20 min. The UV detection wavelength was $245 \mathrm{~nm}$.

\section{Results}

3.1. HPLC Separation of Monosaccharide PMP Derivatives. There have been numerous reports on the separation behavior of PMP-labeled monosaccharides derivatives; however, many of these reports $[18,19]$ were subsequently shown to be unsatisfactory. For example, intermethod and interexperimental variation occur due to the use of different solvents for different solvents and chemicals; the baseline fluctuates widely due to the presence of impurities; rapid and accurate determination cannot be achieved because of the long testing time; the peaks are not sharp enough to enable precise quantitative analysis; and the complexity of the preparation for both eluents can result in poor reproducibility. In this study, distilled water was the only solvent used in addition to dissolved PMP; before derivatization, three methanol-etheracetone extraction and centrifugation steps were performed to remove fat-soluble impurities and pigments from the 


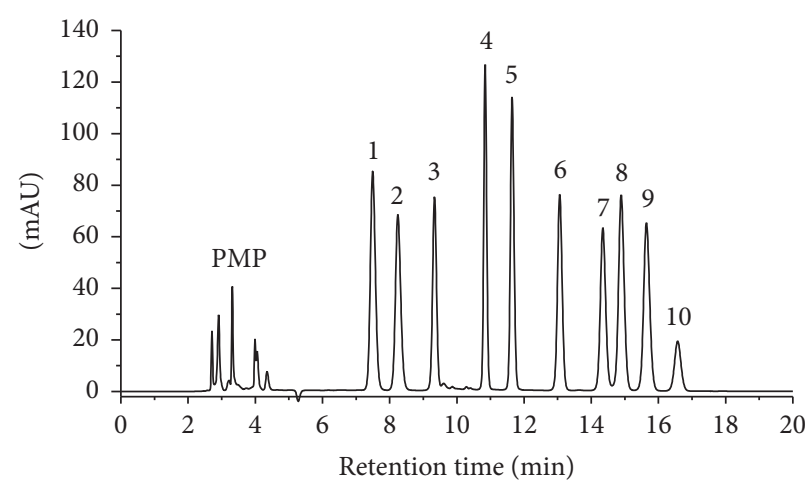

(a)

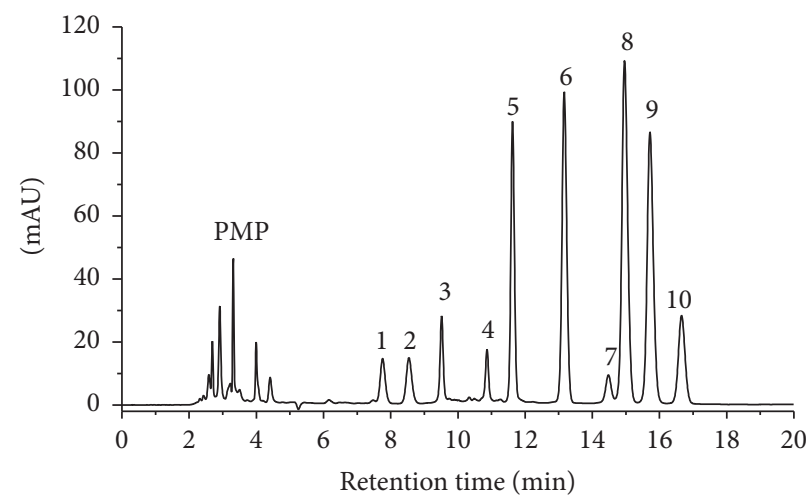

(b)

FIGURE 1: Chromatograms for analysis of (a) the synthetic mixture of ten standard monosaccharides and (b) the component monosaccharides present in Yingshuang green tea TPS using the pre-column PMP derivatization HPLC method. Peaks: 1: mannose; 2: ribose; 3: rhamnose; 4: glucuronic acid; 5: galacturonic acid; 6: glucose; 7: xylose; 8: galactose; 9: arabinose; 10: fucose.

TABLE 1: Retention time, regression analysis, and LOD for the pre-column PMP derivatization HPLC method.

\begin{tabular}{|c|c|c|c|c|c|}
\hline \multirow{2}{*}{ Carbohydrates } & \multirow{2}{*}{ Time (min) } & \multicolumn{2}{|c|}{ Regression equation, $Y=a+b X^{a}$} & \multirow{2}{*}{ Correlation coefficient } & \multirow{2}{*}{ Detection limit $(\mu \mathrm{M})^{\mathrm{b}}$} \\
\hline & & $a$ & $b$ & & \\
\hline Mannose & 6.673 & -0.0496 & 2593.1 & 0.9994 & 0.09 \\
\hline Ribose & 7.292 & -0.0455 & 2268.8 & 0.9994 & 0.10 \\
\hline Rhamnose & 8.244 & -0.0287 & 1875.1 & 0.9993 & 0.19 \\
\hline Glucuronic acid & 10.771 & -0.0957 & 1997.6 & 0.9991 & 0.30 \\
\hline Galacturonic acid & 11.672 & -0.1579 & 2174.9 & 0.9983 & 0.20 \\
\hline Glucose & 12.478 & +0.0139 & 2097.8 & 0.9991 & 0.10 \\
\hline Xylose & 13.678 & -0.0259 & 2577.2 & 0.9980 & 0.07 \\
\hline Galactose & 14.386 & +0.0153 & 2480.6 & 0.9994 & 0.09 \\
\hline Arabinose & 15.12 & +0.0211 & 2436.6 & 0.9994 & 0.10 \\
\hline
\end{tabular}

a: $Y$ and $X$ are the peak area ratio of the analytes to the internal standard (fucose) and concentration of the analytes $(0.2-2 \mu \mathrm{M})$, respectively. b: LOD was defined as the value equal to a signal-to-noise ratio of three.

TPS, thus reducing the baseline fluctuations and protecting the columns; and only eluant B needs to be prepared, thus reducing interexperimental errors. As shown in Figure 1(a), a clear HPLC chromatogram with sharp peaks for each of the ten monosaccharides present in the synthetic mixture was obtained as a result of optimizing the experimental process. Though continually improving the protocol, good baseline separation of the ten monosaccharide derivatives could be achieved within $20 \mathrm{~min}$.

3.2. Validation. Linearity, limit of detection (LOD), reproducibility, and precision were assessed to validate the precolumn PMP derivatization HPLC method. Linearity was assessed by analysis of eight standard sugars (mannose, ribose, rhamnose, glucuronic acid, galacturonic acid, glucose, xylose, galactose, and arabinose; $n=5$ ). The linear regression parameters of the resulting calibration curves are shown in Table 1.

Good linearity (correlation coefficient, $r>0.998$ ) between the peak area ratio of the analytes relative to the internal standard $(Y)$ and the concentration of the standards $(X)$ was achieved over the range of $0.2 \sim 2 \mu \mathrm{mol} / \mathrm{L}$. The LOD for each monosaccharide was determined by analyzing $10 \mu \mathrm{L}$ aliquots of serial dilutions of PMP-derivatized monosaccharide standards prepared using the modified derivatization method. The LOD was defined as the concentration equal to the peak height of a signal-to-noise ratio $(\mathrm{S} / \mathrm{N})$ of three (baseline noise level). The LOD of the individual monosaccharides ranged from 0.09 to $0.2 \mu \mathrm{M}$ (Table 1), indicating that the optimized method is more accurate than previously reported methods.

Furthermore, the precision of the method was assessed by measuring repeatability (intraday variability) and intermediate precision (interday variability) in terms of the retention time and peak area for each monosaccharide standard. The coefficient of variation (CV) for five successive injections of each monosaccharide standard solution $(1 \mu \mathrm{M})$ is presented in Table 2.

The intraday CV values were lower than $0.89 \%$ for the migration time and $3.75 \%$ for the peak areas (A), and the interday $\mathrm{CV}$ values were lower than $1.52 \%$ for the migration time and $4.48 \%$ for the peak areas (A), demonstrating that the precision of the method was similar to previous methods.

3.3. Analysis of TPS from Yingshuang Green Tea. This study was designed to develop a rapid, reproducible, and accurate 
TABLE 2: Intraday and interday precision of the pre-column PMP derivatization HPLC method in terms of the retention time and peak areas of the monosaccharide standards.

\begin{tabular}{lccc}
\hline Carbohydrates & \multicolumn{2}{c}{$\begin{array}{c}\text { Intraday precision }(\mathrm{CV} \%, n=5) \\
\text { Retention time }\end{array}$} & \multicolumn{2}{c}{$\begin{array}{c}\text { Interday precision }(\mathrm{CV} \%, n=5) \\
\text { Retention time }\end{array}$} \\
\hline Mannose & 0.72 & 0.08 & 1.52 \\
Peak area
\end{tabular}

TABLE 3: Determination of the component monosaccharides in Yingshuang green tea TPS and recovery analysis $(n=3)$.

\begin{tabular}{|c|c|c|c|c|c|}
\hline Carbohydrates & Content in sample $(\mu \mathrm{M})$ & Spiked amount $(\mu \mathrm{M})$ & Found amount $(\mu \mathrm{M})$ & Recovery (\%) & RSD (\%) \\
\hline Mannose & 0.28 & 0.50 & 0.76 & 95.2 & 0.7 \\
\hline Ribose & 0.31 & 0.50 & 0.78 & 93.6 & 0.4 \\
\hline Rhamnose & 0.44 & 0.50 & 0.93 & 97.7 & 0.5 \\
\hline Glucuronic acid & 0.28 & 0.50 & 0.75 & 94.7 & 0.8 \\
\hline Galacturonic acid & 0.78 & 0.50 & 1.24 & 91.9 & 1.0 \\
\hline Glucose & 2.00 & 0.50 & 2.51 & 102.4 & 1.2 \\
\hline Xylose & 0.20 & 0.50 & 0.70 & 99.3 & 2.5 \\
\hline Galactose & 1.93 & 0.50 & 2.43 & 98.7 & 0.2 \\
\hline Arabinose & 1.82 & 0.50 & 2.30 & 95.9 & 0.4 \\
\hline
\end{tabular}

analysis method for the quantification of the composition and contents of monosaccharide in TPS isolated from Yingshuang green tea.

The chromatogram for the crude TPS isolated from Yingshuang green tea is shown in Figure 1(b). Each monosaccharide peak was distinct from the baseline and separated from the other peaks, and by comparison with chromatogram of the synthetic mixture of the ten monosaccharide standards, the component monosaccharides present in Yingshuang green tea TPS could be identified. The monosaccharide composition of Yingshuang green tea TPS is summarized in Table 3.

The main monosaccharides present in the crude TPS isolated from Yingshuang green tea were mannose, ribose, rhamnose, glucuronic acid, galacturonic acid, glucose, xylose, galactose, and arabinose, with contents of $0.72,0.78,0.89,0.13$, $0.15,0.36,0.39,0.36,0.36$, and $0.38 \mu \mathrm{M}$, respectively. Neutral glucose, galactose, and arabinose were the predominant monosaccharides in Yingshuang green tea TPS, representing up to $72.1 \%(\mathrm{~mol} \%)$ of total monosaccharides; uronic acids represented $13.0 \%$ of the total carbohydrates in Yingshuang green tea TPS.

Moreover, recovery experiments were performed to assess the accuracy of the method. Known amounts of each monosaccharide standard were individually added to Yingshaung green tea TPS and the samples were measured in triplicate. The recoveries of each of the nine monosaccharides ranged from $93.6 \%$ and $102.4 \%$, and the RSD values were between $0.2 \%$ and $2.5 \%$; these results indicated that the precolumn PMP derivatization HPLC method had a satisfactory sensitivity for the analysis of the monosaccharides present in TPS isolated from Yingshuang green tea.

\section{Discussion}

HPLC represents an effective, quantitative, and comprehensive analysis technique for analysis of carbohydrates. In recent years, methods for the separation of the neutral sugars and uronic acids in polysaccharides have been developed by a number of researchers, but only seven to eight monosaccharides can be successfully separated using these methods, which significantly limits their analytical accuracy $[22,23]$. Pre-column derivatization HPLC has been used to successfully separate mixtures of ten monosaccharides mentioned above, but the analysis was carried out for more than $30 \mathrm{~min}[18,19]$. However in this study, by optimizing previously developed methods to devise a pre-column PMP derivatization HPLC method, good baseline separation of ten monosaccharide derivatives could be obtained within as little as $20 \mathrm{~min}$. Separation of neutral sugars and uronic acids could be achieved with very sharp peaks obtained for each monosaccharide. Furthermore, the recoveries of the component monosaccharides and repeatability of the method described in this study are superior to the method 
devised by Lv's et al. [18]; therefore, our method has a higher accuracy. We propose that method described in this study will be applicable and feasible for monosaccharide composition analysis in food and medicinal plants too as well as general laboratories.

In conclusion, the pre-column PMP derivatization HPLC method described in this paper represents a rapid, accurate, reproducible, cost-effective, and quantitative alternative for the separation of the naturally occurring monosaccharides in TPS isolated from Yingshuang green tea. This method could be applied to analyze the monosaccharide composition and contents in TPS isolated from other tea varieties and may help to explore the relationship between the structure of TPS and their biological activities.

\section{Competing Interests}

The authors declare that they have no competing interests.

\section{Acknowledgments}

This work was supported by National Natural Science Foundation of China (no. 30972402).

\section{References}

[1] D. Wang, Y. Zhao, Y. Sun, and X. Yang, "Protective effects of Ziyang tea polysaccharides on $\mathrm{CCl}_{4}$-induced oxidative liver damage in mice," Food Chemistry, vol. 143, pp. 371-378, 2014.

[2] Z.-Y. Zhao, L.-T. Huangfu, L.-L. Dong, and S.-L. Liu, "Functional groups and antioxidant activities of polysaccharides from five categories of tea," Industrial Crops and Products, vol. 58, pp. 31-35, 2014

[3] Y. Wang, J. Chen, D. Zhang et al., "Tumoricidal effects of a selenium (Se)-polysaccharide from Ziyang green tea on human osteosarcoma U-2 OS cells," Carbohydrate Polymers, vol. 98, no. 1, pp. 1186-1190, 2013.

[4] C. T. Scoparo, L. M. De Souza, Y. D. Rattmann et al., "Polysaccharides from green and black teas and their protective effect against murine sepsis," Food Research International, vol. 53, no. 2, pp. 780-785, 2013.

[5] Y. Wang, Y. Liu, J. Huo, T. Zhao, J. Ren, and X. Wei, “Corrigendum to 'effect of different drying methods on chemical composition and bioactivity of tea polysaccharides,' International Journal of Biological Macromolecules, vol. 64, p. 468, 2014.

[6] Y. Sun, X. Yang, X. Lu, D. Wang, and Y. Zhao, "Protective effects of Keemun black tea polysaccharides on acute carbon tetrachloride-caused oxidative hepatotoxicity in mice," Food and Chemical Toxicology, vol. 58, pp. 184-192, 2013.

[7] Y. Wang, F. Mao, and X. Wei, "Characterization and antioxidant activities of polysaccharides from leaves, flowers and seeds of green tea," Carbohydrate Polymers, vol. 88, no. 1, pp. 146-153, 2012.

[8] X. Chen, Y. Wang, Y. Wu et al., "Green tea polysaccharideconjugates protect human umbilical vein endothelial cells against impairments triggered by high glucose," International Journal of Biological Macromolecules, vol. 49, no. 1, pp. 50-54, 2011.

[9] M. Mori, Hypolipemics containing polysaccharides from green tea: Japanese Kokai Tokkyo Koho, JP02286620A226, 1990.
[10] C. Takeo, H. Kinugasa, and H. Oosu, "APR 24. Extraction of hypoglycemic from tea," Japanese Kokai Tokkyo Koho, JP04124139(92124139) (C1A6/ K3578), 1992.

[11] Y. Shi, Study on Antioxidant and Hypoglycemic Effects of Tea Polysaccharides, Huazhong Agricultural University, Wuhan, China, 2010.

[12] Y. Shi, Z. Yu, Y. Chen, and D. Ni, "Hypoglycemic effects of two tea polysaccharides," Journal of Huazhong Agricultural University, vol. 2, no. 34, pp. 113-119, 2015.

[13] Y. Zhang, Z. Yu, and H. Zhang, "Analysis of monosaccharides in Radix Rehmanniae by GC," China Journal of Chinese Materia Medica, vol. 34, no. 4, pp. 419-422, 2009.

[14] J. Yan, X. Guo, and X. Li, "TLC to fleetly analyze monosaccharide composition of polysaccharide," Journal of Food Science, vol. 27, no. 12, pp. 603-607, 2006.

[15] A. Meyer, C. Raba, and K. Fischer, "Ion-pair RP-HPLC determination of sugars, amino sugars, and uronic acids after derivatization with p-aminobenzoic acid," Analytical Chemistry, vol. 73, no. 11, pp. 2377-2382, 2001.

[16] B.-H. Cheng, J. Y.-W. Chan, B. C.-L. Chan et al., "Structural characterization and immunomodulatory effect of a polysaccharide HCP-2 from Houttuynia cordata," Carbohydrate Polymers, vol. 103, no. 1, pp. 244-249, 2014.

[17] J. Dai, Y. Wu, S.-W. Chen et al., "Sugar compositional determination of polysaccharides from Dunaliella salina by modified RP-HPLC method of precolumn derivatization with 1-phenyl3-methyl-5-pyrazolone," Carbohydrate Polymers, vol. 82, no. 3, pp. 629-635, 2010.

[18] Y. Lv, X. Yang, Y. Zhao, Y. Ruan, Y. Yang, and Z. Wang, "Separation and quantification of component monosaccharides of the tea polysaccharides from Gynostemma pentaphyllum by HPLC with indirect UV detection," Food Chemistry, vol. 112, no. 3, pp. 742-746, 2009.

[19] D. Ren, Y. Jiao, X. Yang, L. Yuan, J. Guo, and Y. Zhao, "Antioxidant and antitumor effects of polysaccharides from the fungus Pleurotus abalonus," Chemico-Biological Interactions, vol. 237, pp. 166-174, 2015.

[20] H. Kuang, Y. Xia, J. Liang, B. Yang, Q. Wang, and Y. Sun, "Fast classification and compositional analysis of polysaccharides from TCMs by ultra-performance liquid chromatography coupled with multivariate analysis," Carbohydrate Polymers, vol. 84, no. 4, pp. 1258-1266, 2011.

[21] M. G. Sevag, D. B. Lackman, and J. Smolens, "The isolation of the components of streptococcal nucleoproteins in serologically," The Journal of Biological Chemistry, vol. 124, pp. 425-436, 1938.

[22] Q. J. Wang and Y. Z. Fang, "Analysis of sugars in traditional Chinese drugs," Journal of Chromatography B, vol. 812, no. 1-2, pp. 309-324, 2004.

[23] X. Yang, Y. Zhao, Q. Wang, H. Wang, and Q. Mei, "Analysis of the monosaccharide components in Angelica polysaccharides by high performance liquid chromatography," Analytical Sciences, vol. 21, no. 10, pp. 1177-1180, 2005. 

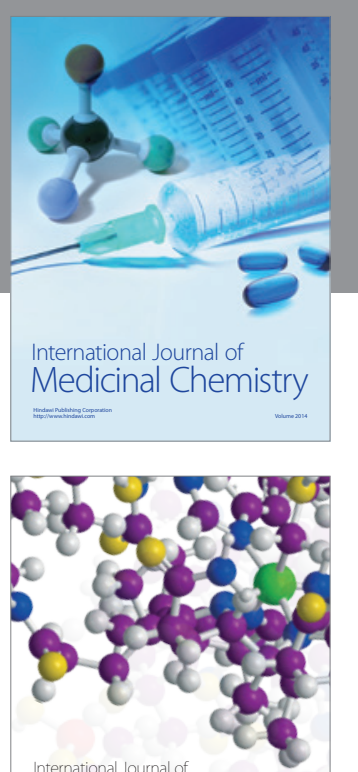

Carbohydrate Chemistry

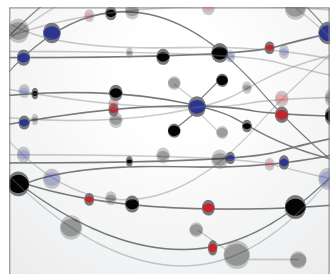

The Scientific World Journal
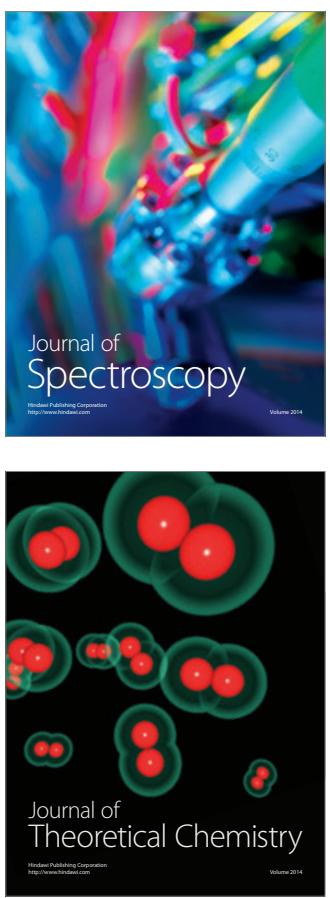
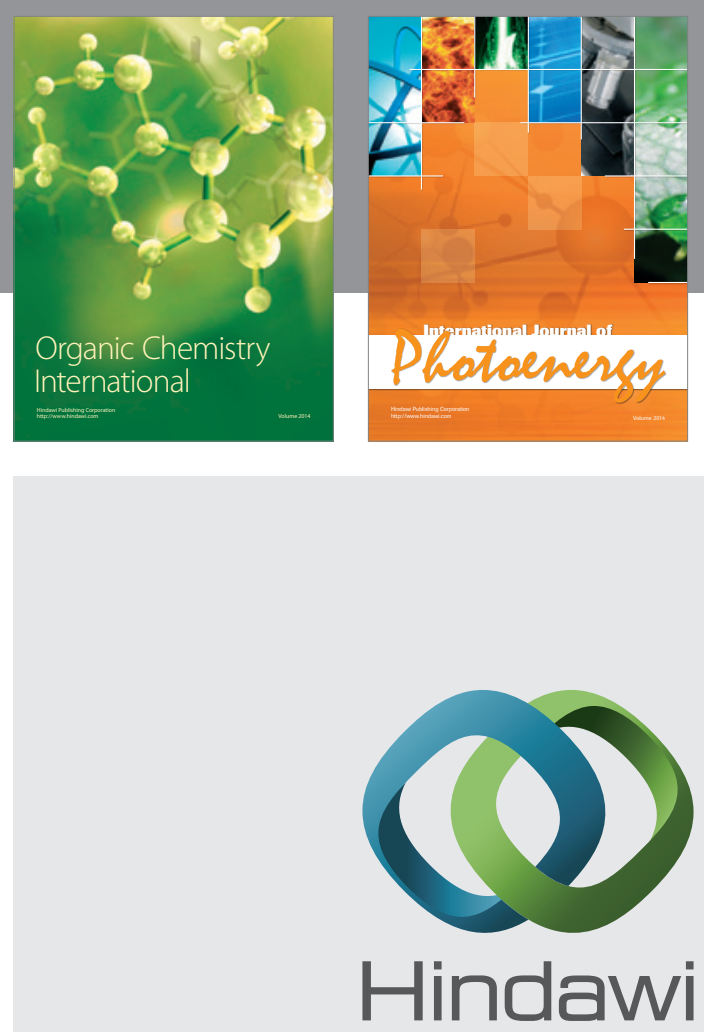

Submit your manuscripts at

http://www.hindawi.com

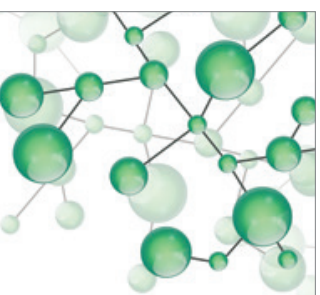

International Journal of

Inorganic Chemistry

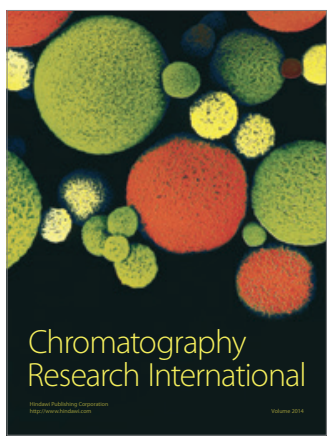

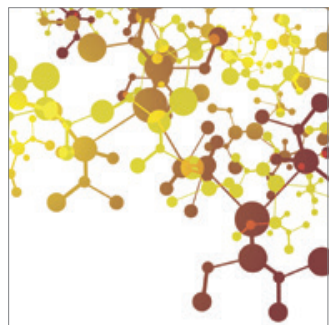

Applied Chemistry
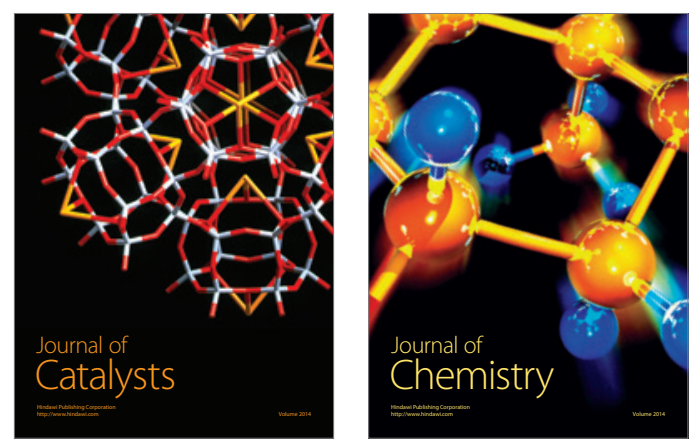
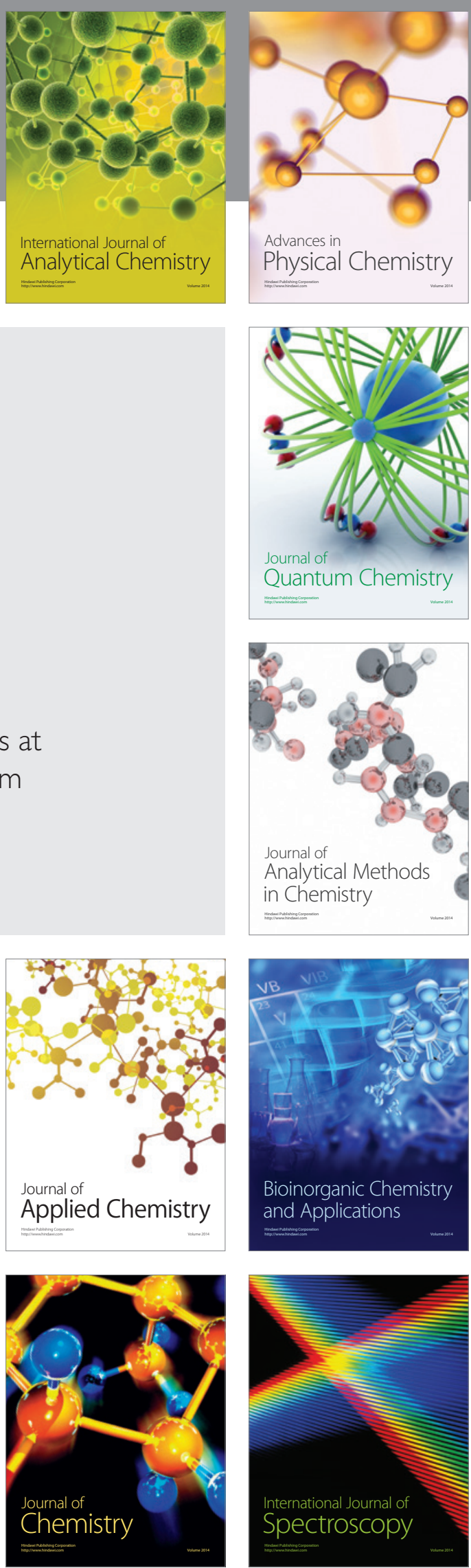